\title{
Ab-initio study of several static and dynamic properties of liquid palladium and platinum.
}

\author{
L. E. González ${ }^{1, *}$, D. J. González ${ }^{1}$, Mohammad Riazuddin Molla ${ }^{2,3}$, A. Z. Ziauddin \\ Ahmed $^{2}$, and G. M. Bhuiyan ${ }^{2}$ \\ ${ }^{1}$ Departamento de Física Teórica, Universidad de Valladolid, Valladolid, Spain \\ ${ }^{2}$ Department of Theoretical Physics, University of Dhaka, Dhaka \\ ${ }^{3}$ Department of Mathematics, University of Dhaka, Dhaka
}

\begin{abstract}
We report a study on several static and dynamic properties of liquid $\mathrm{Pd}$ and $\mathrm{Pt}$ metals at thermodynamic conditions near their respective triple points. The calculations have been carried out by an $a b$ initio molecular dynamics simulation technique. Results are reported for several static structural magnitudes which are compared with the available X-ray diffraction. As for the dynamic properties, results have been obtained for both single and collective dynamical magnitudes as well as for some transport coefficients which are compared with the corresponding experimental data.
\end{abstract}

\section{Introduction.}

Ab-initio molecular dynamics (AIMD) methods based on density functional theory [1] (DFT) are a usual technique for the study of a wide range of condensed matter systems. Most AIMD methods are based on the Kohn-Sham (KS) orbital representation of Density Functional Theory (DFT), whose application poses heavy computational demands and therefore somehow limits the size of the systems under study as well as the simulation times. Nevertheless, this drawback is offset by the very good accuracy provided by these $a b$-initio methods.

This paper reports an AIMD simulation study on several static and dynamic properties of liquid Palladium (l-Pd) and Platinum (l-Pt) at thermodynamic states near their respective triple point. Oddly enough, these two transition metals have not attracted as much theoretical and/or experimental work as most of the other transition metals and, to our knowledge, only a few classical molecular dynamics (CMD) simulations have been carried out on these two liquid metals. Alemany et al [2] have used CMD simulations, combined with interatomic potentials constructed within the framework of the embedded atom model, in order to evaluate the static structure factor, $S(q)$, and some transport coefficients of 1-Pd and l-Pt. A similar calculation was also performed by Kart et al [3], but using a different interatomic potential. On the experimental side, we are only aware of the X-ray diffraction (XD) measurements of Waseda [4], who determined the $S(q)$ of l-Pd and l-Pt near their respective triple points. As for the dynamical structure, no measurements have been performed yet.

*e-mail: luisen@metodos.fam.cie.uva.es 
This dearth of information has prompted us to perform AIMD simulations in order to evaluate several structural and dynamical properties as well as some transport coefficients. We emphasize that this is the first $a b$ initio study on these two metals and, given the superior accuracy of the AIMD versus CMD simulations, we expect these results may stimulate further experimental and/or theoretical work.

In section 2 we briefly describe the theory underlying the present AIMD simulations, and in section 3 we report and discuss some structural and dynamical results which are compared with other previous studies and experimental data. Finally some conclusions are drawn.

\section{Computational method.}

The total potential energy of $N$ ions with valence $Z$, enclosed in a volume $\Omega$, and interacting with $N_{\mathrm{e}}=N Z$ valence electrons is written, within the Born-Oppenheimer (BO) approximation, as the sum of the direct ion-ion coulombic interaction energy, $E_{i-i}\left[\left\{\vec{R}_{l}\right\}\right]$, plus the ground state energy of the electronic system subjected to the ionic external potential created by the ions, $V_{\text {ext }}\left(\left\{\vec{R}_{l}\right\}\right)$,

$$
E\left(\left\{\vec{R}_{l}\right\}\right)=E_{i-i}\left[\vec{R}_{l}\right]+E_{g}\left[n_{g}(\vec{r}), V_{\mathrm{ext}}\left(\left\{\vec{R}_{l}\right\}\right)\right],
$$

where $n_{g}(\vec{r})$ is the ground state electronic density and $\vec{R}_{l}$ are the ionic positions. According to DFT, the ground state electronic density, $n_{g}(\vec{r})$, minimizes the energy functional

$$
E[n(\vec{r})]=T_{s}[n]+E_{\text {ext }}[n]+E_{H}[n]+E_{\mathrm{xc}}[n],
$$

where $T_{s}[n]$ is the non-interacting electronic kinetic energy, $E_{\text {ext }}[n]$ is the electronion interaction energy, $E_{H}[n]$ is the electrostatic Hartree energy and $E_{\mathrm{xc}}[n]$ is the electronic exchange-correlation energy for which we have used the local density approximation [5]. The ion-electron interaction has been described by an ultrasoft pseudopotential [6].

We have performed Kohn-Sham AIMD calculations for l-Pd and l-Pt at thermodynamic conditions near their respective triple point. Specifically, for l-Pd we have studied a thermodynamic state characterized by an ionic number density $\rho=0.0594$ $\AA^{-3}$ and a temperature $T=1873 \mathrm{~K}$ whereas for $1-\mathrm{Pt}$ we used $\rho=0.0577 \AA^{-3}$ and $T=2053 \mathrm{~K}$. For each system, the AIMD simulations were performed by using 120 atoms (i.e. $120 \times(9 d+1 s p)=1200$ valence electrons) in a cubic supercell. Both systems were thermalized during $15.0 \mathrm{ps}$ and therefrom, microcanonical AIMD simulations were performed over 21000 (18500) time steps in l-Pd (l-Pt). As the time step was $0.0055 \mathrm{ps}$ for both systems, the total simulation time amounted to $115.5 \mathrm{ps}$ (l-Pd) and $101.75 \mathrm{ps}(\mathrm{l}-\mathrm{Pt})$.

The configurations were generated under the BO approximation solving the KohnSham equations on a plane-wave basis set for a given configuration of ions, calculating the forces on the ions using the Hellmann-Feynman theorem, and solving the corresponding Newton's equations for constant total energy, as implemented in the Quantum Espresso package [7]. We have used a plane-wave representation with an energy cutoff of $25 \mathrm{Ryd}$ and the single $\Gamma$ point was used in sampling the Brillouin zone. Finally, the 21000 configurations generated for l-Pd (18500 configurations for l-Pt) were used in the evaluation of the corresponding static and dynamic properties. 


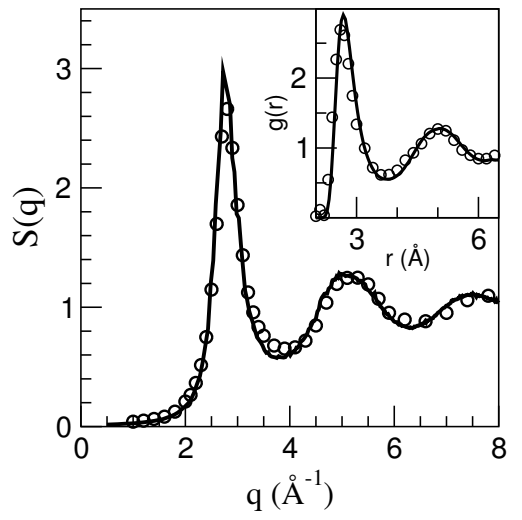

Figure 1. Static structure factor, $S(q)$, of l-Pd at $T=1873 \mathrm{~K}$. Full line: AIMD calculations. Open circles: XD data from Waseda [4]. The inset shows the pair distribution function $g(r)$.

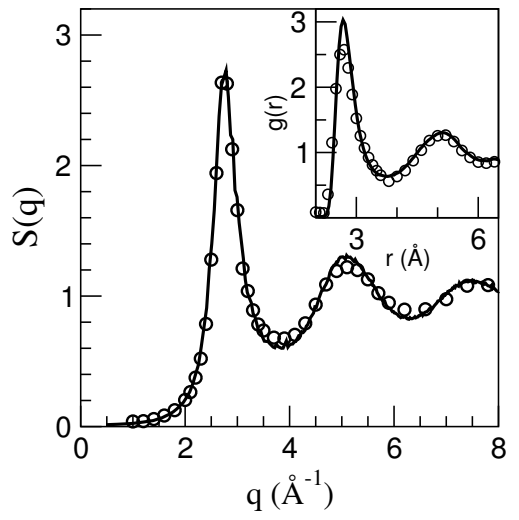

Figure 2. Same as the previous figure, but for l-Pt at $T=2053 \mathrm{~K}$.

\section{Results and discussion.}

\subsection{Static properties}

The AIMD simulations provide a direct evaluation of both the static structure factor, $S(q)$, and the pair distribution function, $g(r)$. Figure 1 shows the AIMD result for the $S(q)$ of l-Pd along with the corresponding XD data [4]. The calculated $S(q)$ has a symmetric main peak at $q_{p}=2.79 \AA^{-1}$, whose shape and amplitude practically coincide with experiment. As for the subsequent oscillations, we observe a very small mismatch. The AIMD obtained $S(q)$ of l-Pt is depicted in Figure 2 and shows an excellent agreement with the XD data [4]. There is a symmetric main peak at $q_{p}=$ $2.77 \AA^{-1}$ and the following oscillations closely follow the experimental ones.

A similar good agreement is found for the AIMD calculated $g(r)$. The coordination number $(\mathrm{CN})$ has been evaluated by integrating the $g(r)$ up to the position of its first minimum, which for both l-Pd and l-Pt stands at the same value $R_{\min }=3.77 \AA$, yielding an identical result of $\mathrm{CN} \approx 12.8$ atoms, which is a typical value of the simple liquid metals near their triple point [8].

We have estimated the isothermal compressibility, $\kappa_{T}$, by resorting to the relation $S(q \rightarrow 0)=\rho k_{B} T \kappa_{T}$ where $k_{B}$ is Boltzmann's constant. The AIMD $S(q)$ has been extrapolated to $q \rightarrow 0$, using $S(q)=s_{0}+s_{2} q^{2}$, yielding $S(q \rightarrow 0)=0.013 \pm 0.001$ for l-Pd and $0.0095 \pm 0.0010$ for l-Pt (the uncertainties have been estimated from the results obtained using different $q$-ranges in the fits). This leads to the values $\kappa_{T}=4.70 \pm 0.20(\mathrm{l}-\mathrm{Pd})$ and $1.80 \pm 0.15\left(\mathrm{l}-\mathrm{Pt}\right.$ ) (in units of $10^{11} \mathrm{~m}^{2} \mathrm{~N}^{-1}$ ). Comparison with experiment, can only be performed for l-Pt for which there is a experimental data, $\kappa_{T} \approx 0.84 \pm 0.02[9]$. 


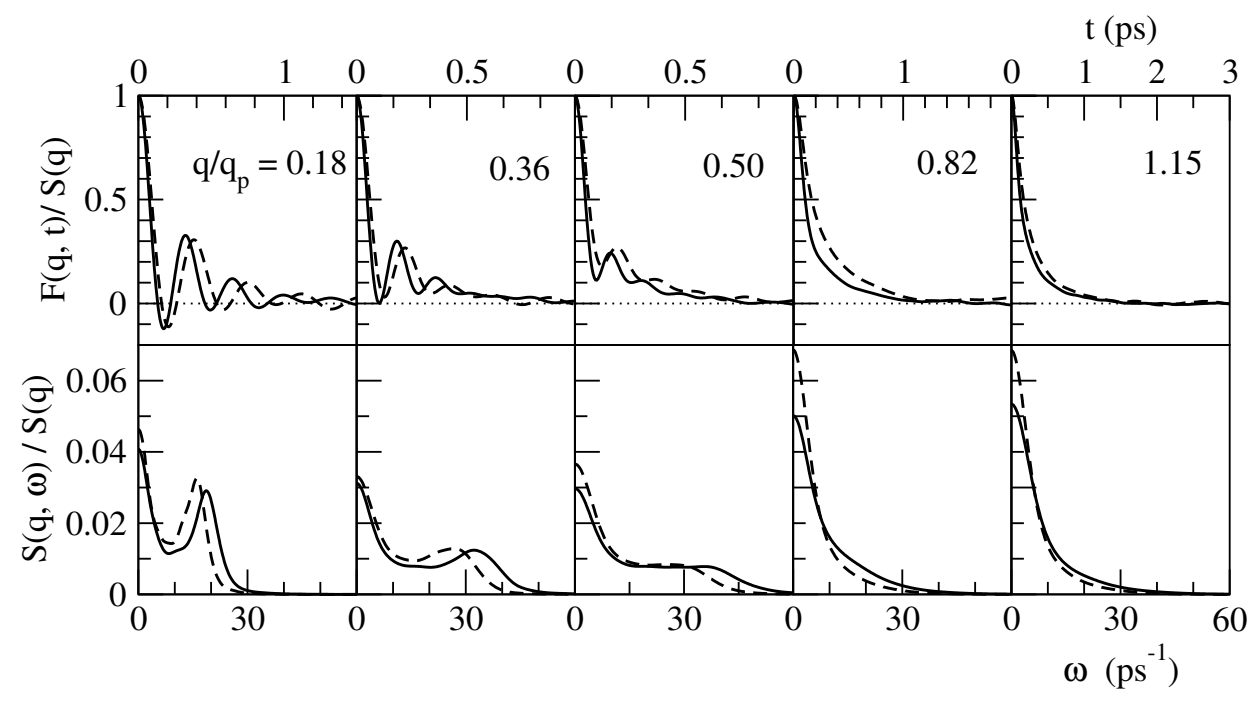

Figure 3. Upper part: Intermediate scattering functions, $F(q, t)$, of 1-Pd at $T=1873 \mathrm{~K}$ (full lines) and l-Pt at $T=2053 \mathrm{~K}$ (dashed lines) at several values of $q / q_{p}$. Lower part: Same as before but for the dynamic structure factors, $S(q, \omega)$

\subsection{Dynamic properties}

\subsubsection{Collective dynamics}

The collective dynamics of density fluctuations in a liquid is described by the intermediate scattering function, $F(q, t)$, defined as

$$
F(q, t)=\frac{1}{N}\left\langle\left(\sum_{j=1}^{N} e^{-i \vec{q} \cdot \vec{R}_{j}\left(t+t_{0}\right)}\right)\left(\sum_{l=1}^{N} e^{i \vec{q} \cdot \vec{R}_{l}\left(t_{0}\right)}\right)\right\rangle,
$$

where $\langle\ldots\rangle$ stands for the ensemble average. Fourier Transform (FT) of the $F(q, t)$ into the frequency domain leads to the dynamic structure factor, $S(q, \omega)$. Another magnitude associated with the density fluctuations is the current due to the overall motion of the particles, i.e.

$$
\vec{j}(q, t)=\sum_{j=1}^{N} \vec{v}_{j}(t) \exp \left[i \vec{q} \cdot \vec{R}_{j}(t)\right]
$$

where $\vec{v}_{j}(t)$ is the velocity of particle $j$ at time $t$. The current is usually split into longitudinal $\left(\vec{j}_{L}(q, t)\right)$ and transverse $\left(\vec{j}_{T}(q, t)\right)$ components with respect to $\vec{q}$. Therefrom, the longitudinal, $C_{L}(q, t)$, and transverse $C_{T}(q, t)$, current correlation functions are obtained as

$$
C_{L}(q, t)=\frac{1}{N}\left\langle\vec{j}_{L}(q, t) \cdot \vec{j}_{L}^{*}(q, 0)\right\rangle \quad C_{T}(q, t)=\frac{1}{2 N}\left\langle\vec{j}_{T}(q, t) \cdot \vec{j}_{T}^{*}(q, 0)\right\rangle
$$

and the time FT gives the associated spectra, $C_{L}(q, \omega)$ and $C_{T}(q, \omega)$ respectively. 


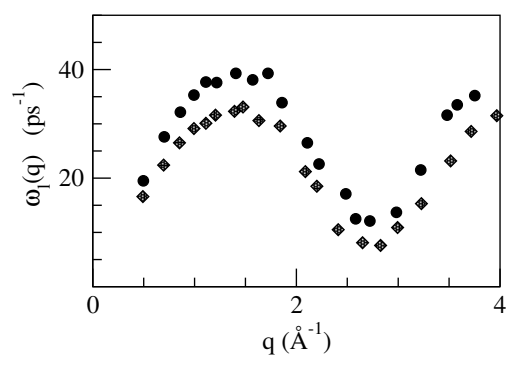

Figure 4. Longitudinal dispersion relation for l-Pd at $T=1873 \mathrm{~K}$ (circles) and for l-Pt at $T=2053 \mathrm{~K}$ (diamonds).

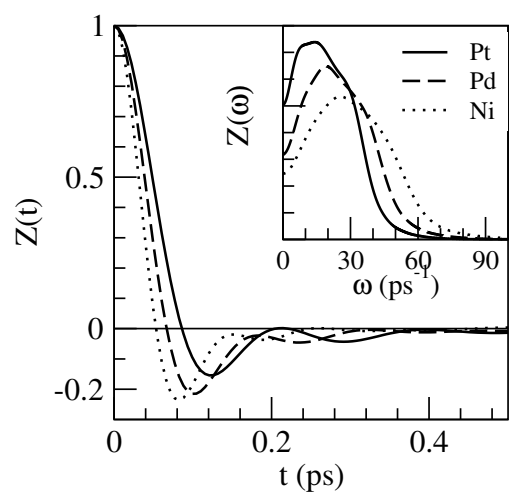

Figure 5. Normalized AIMD calculated velocity autocorrelation function of $1-\mathrm{Pd}$, $\mathrm{l}-\mathrm{Pt}$ and $\mathrm{l}-\mathrm{Ni}$ near their respective melting points. The inset represents the corresponding power spectrum $Z(\omega)$.

Figure 3 depicts, for some $q / q_{p}$-values, the AIMD results for $F(q, t)$ and $S(q, \omega)$ in both l-Pd and l-Pt. For small $q$-values, the $F(q, t)$ show the typical oscillatory behaviour which lasts up to $q \approx(3 / 5) q_{p}$, with the amplitude of the oscillations becoming weaker with increasing $q$-values. Moreover, these oscillations are superposed on a discernible diffusive component. At $q \approx q_{p}$, the $F(q, t)$ exhibit the typical slow decay, known as "de Gennes narrowing".

The associatted $S(q, \omega)$ show side-peaks, indicative of collective density excitations, up to $q \approx(3 / 5) q_{p}$; therefrom the side-peaks evolve into shoulders which last until $q \approx(4 / 5) q_{p}$ and for greater $q$ 's the corresponding $S(q, \omega)$ show a monotonic decreasing behavior.

We have used Eqs. 4-5 to evaluate the current correlation functions $C_{L, T}(q, t)$ and their spectra $C_{L, T}(q, \omega)$. Our AIMD calculated $C_{L}(q, \omega)$ show a peak for each $q$-value and from the frequencies of those peaks, the dispersion relation for the longitudinal modes, $\omega_{l}(q)$, has been obtained and is plotted in Figure 4.

In the low- $q$ region, the slope of the $\omega_{l}(q)$ curve gives a $q$-dependent adiabatic sound velocity that in the limit $q \rightarrow 0$ reduces to the bulk adiabatic sound velocity, $c_{s}$. Using the $q \leq 1.2 \AA^{-1}$ values of our calculated $\omega_{l}(q)$, we have evaluated its slope at $q \rightarrow 0$ and obtained $c_{s} \approx 3450 \pm 150 \mathrm{~m} / \mathrm{s}(\mathrm{l}-\mathrm{Pd})$ and $c_{s} \approx 3000 \pm 150 \mathrm{~m} / \mathrm{s}(\mathrm{l}-\mathrm{Pt})$. The AIMD result for l-Pt compares well with the experimental data, $c_{s, \exp } \approx 3053 \pm$ $150 \mathrm{~m} / \mathrm{s}$ [9].

The transverse current correlation function, $C_{T}(q, t)$, provides information on the shear modes. Its spectrum, $C_{T}(q, \omega)$, when plotted as a function of $\omega$, may display peaks, within some $q$-range, which are related to propagating shear waves. For both l-Pd and l-Pt, we have found that the $C_{T}(q, \omega)$ already shows a peak for the smallest attainable $q$-value, i.e. $q_{\min }=0.18 q_{p}$ and it lasts up to $q \leq 3 q_{p}$, which is typical of the simple liquid metals [8].

From the AIMD results for the $C_{T}(q, t)$, the shear viscosity coefficient, $\eta$, has been calculated as follows $[8,10,11]$. The memory function representation of the $C_{T}(q, t)$ 


$$
\tilde{C}_{t}(q, z)=\frac{1}{\beta m}\left[z+\frac{q^{2}}{\rho m} \tilde{\eta}(q, z)\right]^{-1},
$$

where the tilde denotes the Laplace transform, introduces a generalized shear viscosity coefficient, $\tilde{\eta}(q, z)$. The area under the normalized $C_{T}(q, t)$ gives $\beta m \tilde{C}_{T}(q, z=0)$, wherefrom values for $\tilde{\eta}(q, z=0)$ can be obtained and when extrapolated to $q=0$ give the usual shear viscosity coefficient $\eta$; additional details are given in References $[8,10,11]$. For $1-\mathrm{Pd}$, we have obtained $\eta_{\mathrm{AIMD}} \approx 4.57 \pm 0.15 \mathrm{GPa}$ ps which compares well with the experimental value of $\eta_{\exp }=4.22 \pm 0.15 \mathrm{GPa}$ ps [12]; moreover, the CMD simulation studies have yielded smaller values, i.e. $\eta \approx 3.68 \pm 0.50 \mathrm{GPa}$ ps [2] and $\approx 2.91 \pm 0.86 \mathrm{GPa}$ ps [3]. As for l-Pt, our result $\eta_{\mathrm{AIMD}} \approx 4.90 \pm 0.25 \mathrm{GPa} \mathrm{ps}$ is close to the smaller of the two available experimental data $\eta_{\exp }=4.82$ and $6.74 \mathrm{GPa}$ ps [12]; moreover, the latter one is closer to the CMD simulation result $\eta \approx 6.08 \pm 0.95$ GPa ps [2].

\subsubsection{Single particle dynamics}

An important magnitude is the normalized velocity autocorrelation function (VACF) of a tagged ion in the fluid, $Z(t)$, defined as

$$
Z(t)=\left\langle\vec{v}_{1}(t) \cdot \vec{v}_{1}(0)\right\rangle /\left\langle v_{1}^{2}\right\rangle
$$

with $\vec{v}_{1}(t)$ being the velocity of a tagged ion in the fluid at time $t$.

Figure 5 depicts the calculated $Z(t)$ along with the respective power spectrum (for the sake of comparison we have also included liquid Ni [13]). The $Z(t)$ exhibit the typical backscattering behavior with a first minimum followed by rather weak oscillations and the $Z(t)$ always remaning negative. Notice that the $Z(t)$ are qualitatively very similar and the minor differences are related to the increasing ionic mass when going from $\mathrm{Ni}$ to $\mathrm{Pt}$.

The self-diffusion coefficient, $D$, can be found by either the time integral of $Z(t)$ or from the slope of the mean square displacement $\delta R^{2}(t) \equiv\left\langle\left|\vec{R}_{1}(t)-\vec{R}_{1}(0)\right|^{2}\right\rangle$. Both routes have yielded the same value which, moreover, coincides for both metals, i.e. $D_{\mathrm{AIMD}}(P d)=D_{\mathrm{AIMD}}(P t)=0.27 \pm 0.02 \AA^{2} / \mathrm{ps}$. Unfortunately, the lack of experimental data implies that comparison can only be performed with the avaliable CMD simulations; thus Alemany et al $[2]$ have obtained $D_{\mathrm{CMD}}(\mathrm{Pd})=0.403 \pm 0.003$ $\AA^{2} / \mathrm{ps}$ and $D_{\mathrm{CMD}}(\mathrm{Pt})=0.281 \pm 0.003 \AA^{2} / \mathrm{ps}$. On the other hand, the CMD study of Kart et al $[3]$ gave $D_{\mathrm{CMD}}(P d)=0.496 \pm 0.006 \AA^{2} / \mathrm{ps}$.

\section{Conclusions.}

An ab-initio molecular dynamics simulation method has been used to calculate some static and dynamic properties of l-Pd and l-Pt near their respective triple point. These two metals have not attracted much attention and therefore there is not much theoretical and/or experimental information to compare with. Concerning the static structure, the $a b$ initio results for the pair distribution function, $g(r)$, and the static structure factor, $S(q)$, are in good agreement with the experimental data. The calculations account for the shape and position of the main peak of $S(q)$ as well as the subsequent peaks. The obtained coordination numbers are typical of the simple liquid metals. 
The intermediate scattering functions, $F(q, t)$, show at small $q$-values, strong oscillations superposed on a distinct diffusive component. The associated dynamic structure factors, $S(q, \omega)$, show side-peaks which are indicative of collective density excitations. The calculated transport coeffcients show good agrement with the available experimental data.

LEG and DJG acknowledge the support of MECD (FIS2014-59279-P) and JCyL (VA104A11-2). MRM acknowledges the financial support of the Ministry of Science and Technology (Bangladesh) under the Bangabandhu Fellowship on Science and ICT.

\section{References}

[1] P. Hohenberg and W. Kohn, Phys. Rev. 136, 864 (1964); W. Kohn and L.J. Sham, Phys. Rev. 140, A1133 (1965).

[2] M.M.G. Alemany, C. Rey and L.J. Gallego, J. Chem. Phys. 109, 5175 (1998); Phys. Rev. B 60, 9208 (1999)

[3] S.O. Kart, M. Tomak, M. Uludogan and T. Cagin, J. Non-Cryst. Solids, 337, 101 (2004).

[4] Y. Waseda, The Structure of Non-Crystalline Materials, (New York: McGrawHill, 1980).

[5] D.M. Ceperley and B.J. Alder, Phys. Rev. Letters 45, 566 (1980); J.P. Perdew and A. Zunger, Phys. Rev. B 23, 5048 (1981).

[6] D. Vanderbilt, Phys. Rev. B 41, 7892 (1990)

[7] S. Baroni, A. Dal Corso, S. de Gironcoli, P. Giannozzi, C. Cavazzoni, G. Ballabio, S. Scandolo, G. Chiarotti, P. Focher, A. Pasquarello, K. Laasonen, A. Trave, R. Car, N. Marzari, A. Kokalj, http://www.pwscf.org/

[8] U. Balucani and M. Zoppi, Dynamics of the Liquid State, Clarendon, Oxford, 1994, pag. 261

[9] S. Blairs, Intern. Materials Rev. 52, 321 (2007); Phys. Chem. Liq. 45, 399 (2007)

[10] B.J. Palmer, Phys. Rev. E 49, 359 (1994)

[11] U. Balucani, J.P. Brodholt, P. Jedlovszky and R. Vallauri, Phys. Rev. E 62, 2971 (2000).

[12] T. Iida and R.I.L. Guthrie, The Thermophysical Properties of Metallic Liquids (Oxford Univ. Press, 2015)

[13] B. G. del Rio, L. E. González and D. J. González, these proceedings. 